Publication List

Juntendo Medical Journal

2017. 63 (3), 204-221

\title{
Publications from Juntendo University Graduate School of Medicine, 2015 [1/6]
}

\section{Microbiology}

$\langle$ Orginal Articles〉

* 1) Ohsawa H, Baba T, Enami J, Hiramatsu K: Successful selection of an infection-protective anti-Staphylococcus aureus monoclonal antibody and its protective activity in murine infection models. Microbiol Immunol, 2015; 59: $183-192$.

2) Matsuo M, Hishinuma T, Katayama Y, Hiramatsu K: A mutation of RNA polymerase $\beta$ ' subunit (RpoC) converts heterogeneously vancomycin-intermediate Staphylococcus aureus (hVISA) into "slow VISA". Antimicrob Agents Chemother, 2015; 59: 4215-4225.

3) Ishii K, Tabuchi F, Matsuo M, Tatsuno K, Sato T, Okazaki M, Hamamoto H, Matsumoto Y, Kaito C, Aoyagi T, Hiramatsu K, Kaku M, Moriya K, Sekimizu K: Phenotypic and genomic comparisons of highly vancomycinresistant Staphylococcus aureus strains developed from multiple clinical MRSA strains by in vitro mutagenesis. Sci Rep, 2015; 5: 17092.

4) Uehara Y, Ito T, Ogawa Y, Hirotaki S, Shoji T, Tame T, Horikoshi Y, Hiramatsu K: Molecular epidemiologic study of community-associated methicillin-resistant Staphylococcus aureus with Panton-Valentine leukocidin gene among family members in Japan. J Infect Chemother, 2015; 21: 700-702.

5) Morimoto Y, Baba T, Sasaki T, Hiramatsu K: Apigenin as an anti-quinolone-resistance antibiotic. Int J Antimicrob Agents, 2015; 46 : 666-673.

6) Singh M, Sasaki T, Matsuo M, Morimoto Y, Aiba Y, Hiramatsu K: Complete genome sequence of the drug-naive classical Staphylococcus aureus strain FDA209P. Genome
Announc, 2015; 3: e01343-15.

7) Sasaki T, Tsubakishita S, Kuwahara-Arai K, Matsuo M, Lu YJ, Tanaka Y, Hiramatsu K: Complete genome sequence of methicillinresistant Staphylococcus schleiferi strain TSCC54 of canine origin. Genome Announc, 2015; 3: e01268-15.

8) Fudo S, Yamamoto N, Nukaga M, Odagiri T, Tashiro M, Neya S, Hoshino T: Structural and computational study on inhibitory compounds for endonuclease activity of influenza virus polymerase. Bioorg Med Chem, 2015; 23: $5466-5475$.

9) Sugimura T, Takahashi H, Jounai K, Ohshio K, Kanayama M, Tazumi K, Tanihata Y, Miura Y, Fujiwara D, Yamamoto N: Effects of oral intake of plasmacytoid dendritic cells-stimulative lactic acid bacterial strain on pathogenesis of influenza-like illness and immunological response to influenza virus. $\mathrm{Br}$ J Nutr, 2015; 114: 727-733.

10) Tanaka Y, Sasaki T, Matsuda R, Uematsu Y, Yamaguchi T: Molecular epidemiological study of feline coronavirus strains in Japan using RT-PCR targeting nsp14 gene. BMC Vet Res, 2015; 11: 57.

11) Tanaka Y, Sato Y, Takahashi D, Matsumoto H, Sasaki T: Treatment of a case of feline infectious peritonitis with cyclosporin A. Vet Rec Case Rep, 2015; 3: e000134.

12) Tanaka Y, Amano A, Morisaki M, Sato Y, Sasaki T: Cellular peptidyl-prolyl cis/trans isomerase Pin1 facilitates replication of feline coronavirus. Antiviral Res, 2015; 126: 1-7.

$\langle$ Reviews〉

1) Yamamoto $\mathrm{N}$ : An overview of Ebola virus disease: History, pathogenesis, and treatment. Juntendo Medical Journal, 2015; 61: 396-406. 


\section{Infection Control Science}

〈Orginal Articles〉

* 1) Ohsawa H, Baba T, Enami J, Hiramatsu K: Successful selection of an infection-protective anti-Staphylococcus aureus monoclonal antibody and its protective activity in murine infection models. Microbiol Immunol, 2015; 59: $183-192$.

2) Matsuo M, Hishinuma T, Katayama $Y$, Hiramatsu K: A mutation of RNA polymerase $\beta^{\prime}$ subunit (RpoC) converts heterogeneously vancomycin-intermediate Staphylococcus aureus (hVISA) into "slow VISA". Antimicrob Agents Chemother, 2015; 59: 4215-4225.

3) Ishii $\mathrm{K}$, Tabuchi $\mathrm{F}$, Matsuo M, Tatsuno K, Sato T, Okazaki M, Hamamoto H, Matsumoto Y, Kaito C, Aoyagi T, Hiramatsu K, Kaku M, Moriya K, Sekimizu K: Phenotypic and genomic comparisons of highly vancomycinresistant Staphylococcus aureus strains developed from multiple clinical MRSA strains by in vitro mutagenesis. Sci Rep, 2015; 5: 17092.

4) Uehara Y, Ito T, Ogawa Y, Hirotaki S, Shoji T, Tame T, Horikoshi Y, Hiramatsu K: Molecular epidemiologic study of community-associated methicillin-resistant Staphylococcus aureus with Panton-Valentine leukocidin gene among family members in Japan. J Infect Chemother, 2015; 21: 700-702.

5) Morimoto Y, Baba T, Sasaki T, Hiramatsu K: Apigenin as an anti-quinolone-resistance antibiotic. Int J Antimicrob Agents, 2015; 46: 666-673

6) Singh M, Sasaki T, Matsuo M, Morimoto $Y$, Aiba Y, Hiramatsu K: Complete genome sequence of the drug-naive classical Staphylococcus aureus strain FDA209P. Genome Announc, 2015; 3: e01343-15.

7) Sasaki T, Tsubakishita S, Kuwahara-Arai K, Matsuo M, Lu YJ, Tanaka Y, Hiramatsu K: Complete genome sequence of methicillinresistant Staphylococcus schleiferi strain TSCC54 of canine origin. Genome Announc, 2015; 3: e01268-15.

8) Fudo S, Yamamoto N, Nukaga M, Odagiri T, Tashiro M, Neya S, Hoshino T: Structural and computational study on inhibitory compounds for endonuclease activity of influenza virus polymerase. Bioorg Med Chem, 2015; 23: $5466-5475$.

9) Sugimura T, Takahashi H, Jounai K, Ohshio K, Kanayama M, Tazumi K, Tanihata Y, Miura Y, Fujiwara D, Yamamoto N: Effects of oral intake of plasmacytoid dendritic cells-stimulative lactic acid bacterial strain on pathogenesis of influenza-like illness and immunological response to influenza virus. $\mathrm{Br}$ J Nutr, 2015; 114: 727-733.

$\langle$ Reviews〉

1) Yamamoto N: An overview of Ebola virus disease: History, pathogenesis, and treatment. Juntendo Medical Journal, 2015; 61: 396-406.

\section{Department of Tropical Medicine and Parasitology}

$\langle$ Orginal Articles〉

1) Murai K, Culleton R, Hisaoka T, Endo H, Mita T: Global distribution of polymorphisms associated with delayed Plasmodium falciparum parasite clearance following artemisinin treatment: genotyping of archive blood samples. Parasitol Int, 2015; 64: 267-273.

2) Tanabe K, Zollner G, Vaughan JA, Sattabongkot J, Khuntirat B, Honma H, Mita T, Tsuboi T, Coleman R: Plasmodium falciparum: genetic diversity and complexity of infections in an isolated village in western Thailand. Parasitol Int, 2015; 64: 260-266.

3) Tsukahara T, Ogura S, Sugahara T, Sekihara M, Furusawa T, Kondo N, Mita T, Endo H, Hombhanje F: The choice of healthcare providers for febrile children after introducing non-professional health workers in a malaria endemic area in Papua New Guinea. Frontiers in Public Health, 2015; 3: 275.

4) Hashimoto M, Nara T, Enomoto M, Kurebayashi N, Yoshida M, Sakurai T, Mita T, Mikoshiba K: A dominant negative form of inositol 1,4,5-trisphosphate receptor induces metacyclogenesis and increases mitochondrial density in Trypanosoma cruzi. Biochem Biophys Res Commun, 2015; 466: 475-480.

5) Hashimoto M, Morales J, Uemura H, Mikoshiba K, Nara T: A novel method for inducing amastigote-to-trypomastigote transformation 
in vitro in Trypanosoma cruzi reveals the importance of inositol 1, 4, 5-trisphosphate receptor. PLoS One, 2015; 10: e0135726.

6) Taniguchi T, Miyauchi E, Nakamura S, Hirai M, Suzue K, Imai T, Nomura T, Handa T, Okada H, Shimokawa C, Onishi R, Olia A, Hirata J, Tomita H, Ohno H, Horii T, Hisaeda $\mathrm{H}$ : Plasmodium berghei ANKA causes intestinal malaria associated with dysbiosis. Sci Rep, 2015; 5: 15699.

7) Matsuoka H, Tomita H, Hattori R, Arai M, Hirai M: Visualization of malaria parasites in the skin using the luciferase transgenic parasite, plasmodium berghei. Trop Med Health, 2015; 43: 53-61.

8) Siregar JE, Kurisu G, Kobayashi T, Matsuzaki M, Sakamoto K, Mi-ichi F, Watanabe Y, Hirai M, Matsuoka H, Syafruddin D, Marzuki S, Kita K: Direct evidence for the atovaquone action on the Plasmodium cytochrome bc1 complex. Parasitol Int, 2015; 64: 295-300.

9) Hikosaka K, Hirai M, Komatsuya K, Ono Y, Kita K: Lactate retards the development of erythrocytic stages of the human malaria parasite Plasmodium falciparum. Parasitol Int, 2015; 64: 301-303.

10) Tachibana S-I, Kawai S, Katakai Y, Takahashi H, Nakade T, Yasutomi Y, Horii T, Tanabe K: Contrasting infection susceptibility of the Japanese macaques and cynomolgus macaques to closely related malaria parasites, Plasmodium vivax and Plasmodium cynomolgi. Parasitol Int, 2015; 64: 274-281.

11) Chan CW, Sakihama N, Tachibana S-I, Idris ZM, Lum JK, Tanabe K, Kaneko A: Plasmodium vivax and Plasmodium falciparum at the crossroads of exchange among islands in Vanuatu: implications for malaria elimination strategies. PLoS One, 2015; 10: e0119475.

12) Tachibana S-I, Touhara K, Ejima A: Modification of male courtship motivation by olfactory habituation via the GABAA receptor in Drosophila melanogaster. PLoS One, 2015; 10: e0135186.

13) Lin JW, Spaccapelo R, Schwarzer E, Sajid M, Annoura T, Deroost K, Ravelli RB, Aime E, Capuccini B, Mommaas-Kienhuis AM, O'Toole T, Prins F, Franke-Fayard BM, Ramesar J, Chevalley-Maurel S, Kroeze H,
Koster AJ, Tanke HJ, Crisanti A, Langhorne J, Arese P, Van den Steen PE, Janse CJ, Khan SM: Replication of Plasmodium in reticulocytes can occur without hemozoin formation, resulting in chloroquine resistance. J Exp Med, 2015; 212: 893-903.

14) De Niz M, Helm S, Horstmann S, Annoura T, Del Portillo HA, Khan SM, Heussler VT: In vivo and in vitro characterization of a Plasmodium liver stage-specific promoter. PLoS One, 2015; 10: e0123473.

15) Sugiyama H, Shibata K, Kawakami Y, Arakawa K, Morishima Y, Yamasaki H, Gokuden M, Iwakiri T, Fukumori J: Paragonimiasis due to the consumption of wild boar meat in Japan: Contamination levels of lung fluke larvae in muscle samples of wild boars caught in Kagoshima Prefecture. Jpn J Infect Dis, 2015; 68: 536-537.

16) Lim PK, Yamasaki H, Mak JW, Wong SF, Chong CW, Yap IK, Ambu S, Kumarasamy V: Field evaluation of a rapid diagnostic test to detect antibodies in human toxocariasis. Acta Trop, 2015; 148: 32-37.

17) Weitzel T, Sugiyama H, Yamasaki H, Ramirez C, Rosas R, Mercado R: Human infections with Pseudoterranova cattani nematodes, Chile. Emerg Infect Dis, 2015; 21: 1874-1875. $\langle$ Reviews〉

1) Nara T, Miura S: Current situation of Chagas disease in non-endemic countries. Juntendo Medical Journal, 2015; 61: 389-395.

2) Hirai M, Mita T: Challenging malaria control: do our new genetic tools pave the way for malaria eradication? Juntendo Medical Journal, 2015; 61: 370-377.

3) Mita T: Current research topics in Tropical disease; toward the successful control and elimination; Introduction. Juntendo Medical Journal, 2015; 61: 358-359.

4) Mita T, Jombart T: Patterns and dynamics of genetic diversity in Plasmodium falciparum: what past human migrations tell us about malaria. Parasitol Int, 2015; 64: 238-243.

\section{Host Defense and Biochemical Research}

〈Orginal Articles〉

1) Iba T, Hashiguchi N, Nagaoka I, Tabe $Y$, Kadota K, Sato K: Heparins attenuated 
histone-mediated cytotoxicity in vitro and improved the survival in a rat model of histone-induced organ dysfunction. Intensive Care Med Exp, 2015; 3: 36.

2) Hosoda H, Tamura H, Nagaoka I: Evaluation of the lipopolysaccharide-induced transcription of human TREM-1 gene in vitamin D3-matured THP-1 macrophage-like cells. Int J Mol Med, 2015; 36: 1300-1310.

3) Miyamoto M, Takano M, Iwaya K, Shinomiya N, Goto T, Kato M, Suzuki A, Aoyama T, Hirata J, Nagaoka I, Tsuda H, Furuya K: High-temperature-required protein A2 as a predictive marker for response to chemotherapy and prognosis in patients with highgrade serous ovarian cancers. Br J Cancer, 2015; 112: 739-744.

4) Nagura N, Komatsu J, Iwase H, Hosoda $H$, Ohbayashi O, Nagaoka I, Kaneko K: Effects of the combination of vitamin $\mathrm{K}$ and teriparatide on the bone metabolism in ovariectomized rats. Biomed Reports, 2015; 3: 295-300.

\section{Molecular and Cellular Biochemistory}

$\langle$ Orginal Articles〉

1) Asahara M, Ito N, Yokomizo T, Nakamura M, Shimizu T, Yamada Y: The absence of the leukotriene B4 receptor BLT1 attenuates peripheral inflammation and spinal nociceptive processing following intraplantar formalin injury. Mol Pain, 2015; 11: 11.

2) Hori T, Nakamura M, Yokomizo T, Shimizu T, Miyano M: The leukotriene B4 receptor BLT1 is stabilized by transmembrane helix capping mutations. Biochem Biophys Rep, 2015; 4: 243-249.

3) Okuno T, Ishitani T, Yokomizo T: Biochemical characterization of three BLT receptors in zebrafish. PLoS One, 2015; 10: e0117888.

4) Sawada Y, Honda T, Hanakawa S, Nakamizo S, Murata T, Ueharaguchi-Tanada Y, Ono S, Amano W, Nakajima S, Egawa G, Tanizaki H, Otsuka A, Kitoh A, Dainichi T, Ogawa N, Kobayashi Y, Yokomizo T, Arita M, Nakamura M, Miyachi Y, Kabashima K: Resolvin E1 inhibits dendritic cell migration in the skin and attenuates contact hypersensitivity responses. J Exp Med, 2015; 212: 1921-1930.

5) Zandi S, Nakao S, Chun KH, Fiorina P, Sun D,
Arita R, Zhao M, Kim E, Schueller O, Campbell S, Taher M, Melhorn MI, Schering A, Gatti F, Tezza S, Xie F, Vergani A, Yoshida S, Ishikawa K, Yamaguchi M, Sasaki F, Schmidt-Ullrich R, Hata Y, Enaida H, Yuzawa M, Yokomizo T, Kim YB, Sweetnam P, Ishibashi T, Hafezi-Moghadam A: ROCKisoform-specific polarization of macrophages associated with age-related macular degeneration. Cell Rep, 2015; 10: 1173-1186.

6) Kubota S, Watanabe M, Shirato M, Okuno T, Higashimoto I, Machida K, Yokomizo T, Inoue $\mathrm{H}$ : An inhaled phosphodiesterase 4 inhibitor E6005 suppresses pulmonary inflammation in mice. Eur J Pharmacol, 2015; 768: 41-48.

$\langle$ Reviews〉

1) Liu M, Yokomizo T: The role of leukotrienes in allergic diseases. Allergol Int, 2015: 64: 17-26.

2) Yokomizo T: Two distinct leukotriene $B 4$ receptors, BLT1 and BLT2. J Biochem, 2015: 157: $65-71$.

〈Books〉

1) In: Yokomizo T, Murakami M, eds. Bioactive Lipid Mediators: Current Reviews and Protocols. Tokyo: Springer, 2015.

2) Koga T, Yokomizo, T: Chapter 6: Leukotriene B4 Receptors. In: Yokomizo T, Murakami M, eds. Bioactive Lipid Mediators: Current Reviews and Protocols. Tokyo: Springer, 2015; 85-93.

3) Okuno T, Yokomizo T: Chapter 21: Basic Techniques for Lipid Extraction from Tissues and Cells. In: Yokomizo T, Murakami M, eds. Bioactive Lipid Mediators: Current Reviews and Protocols. Tokyo: Springer, 2015; 331336.

4) Sasaki F, Yokomizo T: Chapter 29: A Novel Anti-FLAG Monoclonal Antibody Useful for GPCR Study. In: Yokomizo T, Murakami M, eds. Bioactive Lipid Mediators: Current Reviews and Protocols. Tokyo: Springer, 2015; 409-417.

\section{Immunology}

\section{〈Orginal Articles〉}

1) Kamachi F, Isshiki T, Harada N, Akiba H, Miyake S: ICOS promotes group 2 innate lymphoid cell activation in lungs. Biochem 
Biophys Res Commun, 2015; 463: 739-745.

2) Miyake S, Kim S, Suda W, Oshima K, Nakamura M, Matsuoka T, Chihara N, Tomita A, Sato W, Kim SW, Morita H, Hattori M, Yamamura T: Dysbiosis in the gut microbiota of patients with multiple sclerosis, with a striking depletion of species belonging to Clostridia XIVa and IV clusters. PLoS One, 2015; 10: e0137429.

3) Croxford JL, Miyake S: Immunoregulation of multiple sclerosis by gut environmental factors. Clinical and Experimental Neuroimmunology, 2015; 6: 362-369.

4) Sato A, Nishida C, Sato-Kusubata K, Ishihara M, Tashiro Y, Gritli I, Shimazu H, Munakata S, Yagita H, Okumura K, Tsuda Y, Okada Y, Tojo A, Nakauchi H, Takahashi S, Heissig B, Hattori K: Inhibition of plasmin attenuates murine graft-versus-host disease mortality by suppressing the matrix metalloproteinase-9-dependent inflammatory cytokine storm and effector cell trafficking. Leukemia, 2015; 29: 145-156.

5) Kato T, Sakata-Yanagimoto M, Nishikii H, Ueno M, Miyake Y, Yokoyama Y, Asabe Y, Kamada Y, Muto H, Obara N, Suzukawa K, Hasegawa Y, Kitabayashi I, Uchida K, Hirao A, Yagita H, Kageyama R, Chiba S: Hes1 suppresses acute myeloid leukemia development through FLT3 repression. Leukemia, 2015; 29: 576-585.

6) Karasawa K, Asano K, Moriyama S, Ushiki M, Monya M, Iida M, Kuboki E, Yagita H, Uchida K, Nitta K, Tanaka M: Vascular-resident CD169-positive monocytes and macrophages control neutrophil accumulation in the kidney with ischemia-reperfusion injury. J Am Soc Nephrol, 2015; 26: 896-906.

* 7) Kato T, Sakata-Yanagimoto M, Nishikii H, Ueno M, Miyake Y, Yokoyama Y, Asabe Y, Kamada Y, Muto H, Obara N, Suzukawa K, Hasegawa Y, Kitabayashi I, Uchida K, Hirao A, Yagita H, Kageyama R, Chiba S: Inhibition of plasmin protects against colitis in mice by suppressing matrix metalloproteinase 9-mediated cytokine release from myeloid cells. Gastroenterology, 2015; 148: 565-578.

8) Maekawa $Y$, Ishifune $C$, Tsukumo $S$, Hozumi K, Yagita H, Yasutomo K: Notch controls the survival of memory CD4 $+\mathrm{T}$ cells by regulating glucose uptake. Nat Med, 2015; 21: 55-61.

9) Bang BR, Kim SJ, Yagita H, Croft M, Kang YJ: Inhibition of 4-1BBL-regulated TLR response in macrophages ameliorates endotoxininduced sepsis in mice. Eur J Immunol, 2015; 45: $886-892$.

10) Lézot F, Chesneau J, Navet B, Gobin B, Amiaud J, Choi Y, Yagita H, Castaneda B, Berdal A, Mueller CG, Rédini F, Heymann D: Skeltal consequences of RANKL-blocking antibody (IK22-5) injections during growth: mouse strain disparities and synergic effect with zoledronic acid. Bone, 2015; 73: 51-59.

* 11) Shindo Y, Yoshimura K, Kuramasu A, Watanabe Y, Ito H, Kondo T, Oga A, Ito H, Yoshino S, Hazama S, Tamada K, Yagita H, Oka M: Combination immunotherapy with 4-1BB activation and PD-1 blockade enhances antitumor efficacy in a mouse model of subcutaneous tumor. Anticancer Res, 2015; 35: $129-136$.

12) Voron T, Colussi O, Marcheteau E, Pernot $S$, Nizard M, Pointet A, Latreche S, Bergaya S, Benhamouda N, Tanchot C, Stockmann C, Combe P, Berger A, Zinzindohoue F, Yagita $\mathrm{H}$, Tartour E, Taieb J, Terme M: VEGF-A modulates expression of inhibitory checkpoints on CD8 + T cells in tumors. J Exp Med, 2015; 212: 139-148.

*13) Doi K, Imai T, Kressler C, Yagita H, Agata Y, Vooijs M, Hamazaki Y, Inoue J, Minato N: Crucial role of the Rap G protein signal in Notch activation and leukemogenicity of T-cell acute lymphoblastic leukemia. Sci Rep, 2015; 5: 7978.

14) McKay JT, Egan RP, Yammani RD, Chen L, Shin T, Yagita H, Haas KM: PD-1 suppresses protective immunity to Streptococcus pneumoniae through a B cell-intrinsic mechanism. J Immunol, 2015; 194: 2289-2299.

15) McAlees JW, Lajoie S, Dienger K, Sproles AA, Richgels PK, Yang Y, Khodoun M, Azuma M, Yagita H, Fulkerson PC, WilsKarp M, Lewkowich IP: Differential control of $\mathrm{CD} 4+\mathrm{T}$-cell subsets by the PD-1/PD-L1 axis in a mouse model of allergic asthma. Eur J Immunol, 2015; 45: 1019-1029.

16) Matsumura T, Sugawara Y, Yutani M, 
Amatsu S, Yagita H, Kohda T, Fukuoka S, Nakamura Y, Fukuda S, Hase K, Ohno H, Fujinaga Y: Botulinum toxin A complex exploits intestinal $\mathrm{M}$ cells to enter the host and exert neurotoxicity. Nat Commun, 2015; 6: 6255 .

17) Blake SJ, Ching AL, Kenna TJ, Galea R, Large J, Yagita H, Steptoe RJ: Blockade of PD-1/ PD-L1 promotes adoptive T-cell immunotherapy in a tolerogenic environment. PLoS One, 2015; 10: e0119483.

18) Pedrosa AR, Trindade A, Fernandes AC, Carvalho C, Gigante J, Tavares AT, DiéguezHurtado R, Yagita H, Adams RH, Duarte A: Endothelial Jagged1 antagonizes Dll4 regulation of endothelial branching and promotes vascular maturation downstream of Dll4/ Notch1 Arterioscler. Thromb Vasc Biol, 2015; 35: 1134-1146.

19) Dhainaut M, Coquerelle $C$, Uzureau $S$, Denoeud J, Acolty V, Oldenhove G, Galuppo A, Sparwasser T, Tielemans K, Pays E, Yagita H, Borst J, Moser M: Thymusderived regulatory $\mathrm{T}$ cells restrain pro- $^{-}$ inflammatory Th1 responses by downregulating CD70 on dendritic cells. EMBO J, 2015; 34: $1336-1348$.

20) Nakano N, Nishiyama C, Yagita H, Hara M, Motomura Y, Kubo M, Okumura K, Ogawa H: Notch signaling enhances FceRI-mediated cytokine production by mast cells through direct and indirect mechanisms. J Immunol, 2015; 194: 4535-4544.

21) Tripathi S, Chabtini L, Dakle PJ, Smith B, Akiba H, Yagita H, Guleria I: Effect of TIM-3 blockade on the immunophenotype and cytokine profile of murine uterine NK cells. PLoS One, 2015; 10: e0123439.

*22) Nakano Y, Negishi N, Gocho S, Mine T, Sakurai Y, Yazawa M, Abe K, Yagita H, Habu S, Kageyama R, Kawaguchi Y, Hozumi K: Disappearance of centroacinar cells in the Notch ligand-deficient pancreas. Genes Cells, 2015; 20: 500-511.

23) Hirano KI, Negishi N, Yazawa M, Yagita H, Habu S, Hozumi K: Delta-like 4-mediated notch signaling is required for early $\mathrm{T}$-cell development in a three-dimentional thymic structure. Eur J Immunol, 2015; 45: 2252-
2262.

24) Shimada S, Nonomura S, Mori S, Suemizu H, Itoh T, Takebayashi S, Okada Y, Yahata T, Shiina T, Katoh H, Suzuki R, Tani K, Ando K, Yagita H, Habu S, Sasaki E, Kametani Y: Common marmoset CD117-positive hematopoietic cells possess multipotency. Int Immunol, 2015; 27: 567-577.

25) Ooi JD, Li M, Kourkoutzelos K, Yagita H, Azuma M, Holdsworth SR, Kitching AR: PD- 1 and its ligands do not limit experimental foreign antigen induced immune complex glomerulonephritis. Nephrology (Carlton), 2015; 20: 892-898.

26) Li H, Fu YX, Wu Q, Zhou Y, Crossman DK, Yang P, Li J, Luo B, Morel LM, Kabarowski JH, Yagita H, Ware CF, Hsu HC, Mounts JD: Interferon-induced mechanosensing defects impede apoptotic cell clearance in lupus. J Clin Invest, 2015; 125: 2877-2890.

27) Bransi A, Salgado OC, Beffinger M, Milo K, Silina K, Yagita H, Becher B, Knuth A, van den Broek M: Rational conbination of immunotherapies with clinical efficacy in mice with advanced cancer. Cancer Immunol Res, 2015; 3: $1279-1288$.

28) Kakizaki A, Fujimura T, Furudate S, Kambayashi Y, Yamauchi T, Yagita H, Aiba S: Immunomodulatory effect of peritumorally administered interferon-beta on melanoma through tumor-associated macrophages. Oncoimmunology, 2015; 4: e1047584.

29) De Jesus M, Rodriguez AE, Yagita H, Ostroff GR, Mantis NJ: Sampling of Candida albicans and Candida tropicalis by Langerin-positive dendritic cells in mouse Peyer's patches. Immunol Lett, 2015; 168: 64-72.

30) Koga JI, Nakano T, Dahlman JE, Figueiredo JL, Zhang H, Decano J, Khan OF, Niida T, Iwata H, Aster JC, Yagita H, Anderson DG, Ozaki CK, Aikawa M: Macrphage Notch ligand Delta-like 4 promotes vein graft lesion development: Implications for the treatment of vein graft failure. Arterioscler Thromb Vasc Biol, 2015; 35: 2343-2353.

31) Dong H, Franklin NA, Ritchea SB, Yagita H, Glennie MJ, Bullock TN: CD70 and IFN-1 selectively induce Eomesdermin or $\mathrm{T}$-bet and synergize to promote CD8 $+\mathrm{T}$-cell 
responses. Eur J Immunol, 2015; 45: 32893301.

32) Shanker A, Pellom ST Jr, Dudimah DF, Thounaojam MC, de Kluiver RL, Brooks AD, Yagita H, McVicar DW, Murphy WJ, Longo DL, Sayers TJ: Bortezomib improves adoptive $T$ cell therapy by sensitizing cancer cells to FasL cytotoxicity. Cancer Res, 2015; 75: 5260-5272.

33) Sharma MD, Shinde R, McGaha TL, Huang L, Holmgaard RB, Wolchok JD, Mautino MR, Celis E, Sharpe AH, Francisco LM, Powell JD, Yagita H, Mellor AL, Blazar BR, Munn DH: The PTEN pathway in Treg is a critical driver of the suppressive tumor microenvironment. Sci Adv, 2015; 1: e1500845.

* 34) Katsura Y, Harada N, Harada S, Ishimori A, Makino F, Ito J, Kamachi F, Okumura K, Akiba H, Atsuta R, Takahashi K: Characteristics of alveolar macrophages from murine models of OVA-induced allergic airway inflammation and LPS-induced acute airway inflammation. Exp Lung Res, 2015; 41: 370382.

35) Tripathi S, Chabtini L, Dakle PJ, Smith B, Akiba H, Yagita H, Guleria I: Effect of TIM-3 blockade on the immunophenotype and cytokine profile of murine uterine NK cells. PLoS One, 2015; 10: e0123439.

\section{Molecular Pathogenesis}

〈Orginal Articles〉

* 1) Ito Y, Kawano H, Kanai F, Nakamura E, Tada $\mathrm{N}$, Takai S, Horie S, Arai H, Kobayashi T, Hino O: Establishiment of Tsc2-deficient rat embryonic stem cells. Int J Onco, 2015; 46: 1944-1952.

2) Saeki H, Suzuki C, Yamasaki S, Hashizume A, Izumi H, Suzuki F, Ishi K, Nojima M, Hino O: Cotyledonoid dissecting leiomyoma of the uterus: report of two case. Arch Gynecol Obstet, 2015; 291: 357-361.

3) Sugiura H, Yasuda S, Katsurabayashi S, Kawano H, Endo K, Takasaki K, Iwasaki K, Ichikawa M, Kobayashi T, Hino O, Yamagata $\mathrm{K}$ : Rheb actvation disrupts spine synapse formation through accumulation of syntenin in tuberous sclerosis complex. Nat Commun, 2015; 6: 6842 .
4) Horimoto $Y$, Arakawa A, Tanabe M, Kuroda K, Matsuoka J, Igari F, Himuro T, Yoshida Y, Tokuda E, Shimizu H, Hino O, Saito M: Menstrual cycle could affect Ki67 expression in estrogen receptor-positive breast cancer patients. J Clin Pathol, 2015; 68: 825-829.

* 5) Kawano H, Ito Y, Kanai F, Nakamura E, Tada N, Takai S, Horie S, Kobayashi T, Hino O: Aberrant differentiation of Tsc2-deficient teratomas associated with activation of the mTORC1-TFE3 pathway. Oncol Rep, 2015; 34: 2251-2258.

6) Sai S, Vares G, Kim EH, Karasawa K, Wang B, Nenoi M, Horimoto Y, Hayashi M: Carbon ion beam combined with cisplatin effectively disrupts triple negative breast cancer stemlike cells in vitro. Mol Cancer, 2015; 14: 166.

7) Shimizu H, Horimoto Y, Sonoue H, Arakawa A, Kurata M, Kosaka T, Nakai K, Himuro T, Tokuda E, Takahashi Y, Taira F, Ito M, Abe I, Senuma K, Stork-Sloots L, Snoo F, Saito M: Application of a 70-gene expression profile to Japanese breast cancer patients Breast Care (Basel), 2015; 10: 118-122.

8) Horimoto Y, Arakawa A, Harada-Shoji N, Sonoue H, Yoshida Y, Himuro T, Igari F, Tokuda E, Mmat O, Tanabe M, Hino O, Saito M: Low FOXA1 expression predicts good response to neo-adjuvant chemotherapy resulting in good outcomes for luminal HER2-negative breast cancer cases. Br J Cancer, 2015; 112: 345-351.

9) Holmquist E, Reitsma B, King B, EscuderoEsparza Sioned Owen A, Orimo A, Okroj M, Anagnostaki L, Jiang W, Jirström K, Blom A: The human complement inhibitor Sushi Domain-Containing Protein 4 (SUSD4) expression in tumor cells and CD8 $+\mathrm{T}$ cells is associated with better prognosis of breast cancer patients. BMC Cancer, 2015; 15: 737.

10) Miyazaki J, Tsurui H, Kawasumi K, Kobayashi T: Simultaneous dual-wavelength imaging of nonfluorescent tissues with 3D subdiffraction photothermal microscopy. Opt Express, 2015; 23: 3647-3656.

11) He J, Miyazaki J, Wang $N$, Tsurui $H$, Kobayashi T: Biological imaging with nonlinear photothermal microscopy using a compact supercontinuum fiber laser source. Opt 
Express, 2015; 23: 9762-9771.

12) Miyazaki J, Tsurui H, Kobayashi T: Reduction of distortion in photothermal microscopy and its application to the high-resolution three-dimensional imaging of nonfluorescent tissues. Biomed Opt Express, 2015; 6: 32173224.

13) Miyazaki J, Tsurui H, Kawasumi K, Kobayashi T: Sensitivity enhancement of photothermal microscopy with radially segmented balanced detection. Opt Lett, 2015; 40: 479482.

* 14) Ohtsuji M, Lin Q, Nishikawa K, Ohtsuji N, Okazaki H, Tsurui H, Amano H, Shirai T, Nishimoto N, Nishimura H, Hirose S: IL-6 signal blockade ameliorates the enhanced osteoclastogenesis and the associated joint destruction in a novel FcgRIIB-deficient rheumatoid arthritis mouse model. Mod Rheumatol, 2015; 25: 270-277.

〈Reviews〉

1) Imai $M$, Hino $O$ : Environmental carcinogenesis-100th anniversary of creating cancer. Cancer Science, 2015; 106: 1483-1485.

\section{Rheumatology}

〈Orginal Articles〉

1) Doe $K$, Nozawa $K$, Hirai $T$, Tsushima $H$, Hayashi E, Hiruma K, Ando S, Nakano S, Kon T, Amano H, Yamaji K, Tamura N, Takasaki Y: Second-to-fourth digit ratio in systemic lupus erythematosus. J Rheumatol, 2015; 42: 826-828

2) Hara R, Miyazawa H, Nishimura K, Momoi T, Nozawa T, Kikuchi M, Sakurai N, Kizawa T, Shimamura S, Yasuda S, Hiromura K, Sada KE, Kawaguchi Y, Tamura N, Takei S, Takasaki Y, Atsumi T, Mori M: A national survey on current use of mycophenolate mofetil for childhood-onset systemic lupus erythematosus in Japan. Mod Rheumatol, 2015; 25: 858-864.

3) Honda D, Tsueshita K, Ohsawa I, Miyashita $\mathrm{T}$, Inoshita H, Shimamoto M, Horikoshi S, Motimoto S, Takasaki Y, Tomino Y: Clinical significance of renal interstitial fibrosis in patients with lupus nephritis. Juntendo Medical Journal, 2015; 61: 418-425.

4) Hotta A, Kuwatsuru R, Asahi K, Okada S,
Tsuge D, Shiraishi A, Takasaki Y: Transcatheter arterial coil embolization of ruptured common hepatic artery aneurysm in a patient with Behçet's disease. Case Rep Radiol, 2015; 2015: 790175.

5) Ishiyama K, Yashiro T, Nakano N, Kasakura K, Miura R, Hara M, Kawai F, Maeda K, Tamura N, Okumura K, Ogawa H, Takasaki Y, Nishiyama C: Involvement of PU.1 in NFATc1 promoter function in osteoclast development. Allergol Int, 2015; 64: 241-247.

6) Koike H, Akiyama K, Saito T, Sobue G; Research Group for IVIg for EGPA/CSS in Japan: Intravenous immunoglobulin for chronic residual peripheral neuropathy in eosinophilic granulomatosis with polyangiitis (Churg-Strauss syndrome): a multicenter, double-blind trial. J Neurol, 2015; 262: 752759 .

* 7) Matsuki Y, Atsumi T, Yamaguchi K, Hisano M, Arata N, Oku K, Watanabe N, Sago H, Takasaki Y, Murashima A: Clinical features and pregnancy outcome in antiphospholipid syndrome patients with history of severe pregnancy complications. Mod Rheumatol, 2015; 25: 215-218.

8) Nakano S, Morimoto S, Suzuki S, Tsushima H, Yamanaka K, Sekigawa I, Takasaki Y: Immunoregulatory role of IL-35 in $\mathrm{T}$ cells of patients with rheumatoid arthritis. Rheumatology (Oxford), 2015; 54: 1498-1506.

9) Ohtsuji M, Lin Q, Nishikawa K, Ohtsuji N, Okazaki H, Tsurui H, Amano H, Shirai T, Nishimoto N, Nishimura H, Hirose S: IL-6 signal blockade ameliorates the enhanced osteoclastogenesis and the associated joint destruction in a novel FcrRIIB-deficient rheumatoid arthritis mouse model. Mod Rheumatol, 2015; 25: 270-277.

10) Sada KE, Yamamura M, Harigai M, Fujii T, Takasaki Y, Amano K, Fujimoto S, Muso E, Murakawa Y, Arimura Y, Makino H; Research Committee on Intractable Vasculitides, the Ministry of Health, Labour and Welfare of Japan: Different responses to treatment across classified diseases and severities in Japanese patients with microscopic polyangiitis and granulomatosis with polyangiitis: a nationwide prospective 
inception cohort study. Arthritis Res Ther, 2015; 17: 305.

11) Sakai R, Cho SK, Nanki T, Watanabe $K$, Yamazaki H, Tanaka M, Koike R, Tanaka Y, Saito K, Hirata S, Amano K, Nagasawa H, Sumida T, Hayashi T, Sugihara T, Dobashi H, Yasuda S, Sawada T, Ezawa K, Ueda A, Fujii T, Migita K, Miyasaka N, Harigai M: REAL Study Group: Head-to-head comparison of the safety of tocilizumab and tumor necrosis factor inhibitors in rheumatoid arthritis patients (RA) in clinical practice: results from the registry of Japanese RA patients on biologics for long-term safety (REAL) registry. Arthritis Res Ther, 2015; 17: 74.

12) Suzuki S, Morimoto S, Fujishiro M, Kawasaki M, Hayakawa K, Miyashita T, Ikeda K, Miyazawa K, Yanagida M, Takamori K, Ogawa H, Sekigawa I, Takasaki Y: Inhibition of the insulin-like growth factor system is a potential therapy for rheumatoid arthritis. Autoimmunity, 2015; 48: 251-258.

13) Tanaka M, Koike R, Sakai R, Saito K, Hirata S, Nagasawa H, Kameda H, Hara M, Kawaguchi Y, Tohma S, Takasaki Y, Dohi M, Nishioka Y, Yasuda S, Miyazaki Y, Kaneko Y, Nanki T, Watanabe K, Yamazaki H, Miyasaka N, Harigai M: Pulmonary infections following immunosuppressive treatments during hospitalization worsen the short-term vital prognosis for patients with connective tissue disease-associated interstitial pneumonia. Mod Rheumatol, 2015; 25: 609-614.

14) Tokai N, Ogasawara M, Gorai M, Matsuki $Y$, Yamada Y, Murayama G, Sugisaki N, Nemoto T, Ando S, Minowa K, Kon T, Tada K, Matsushita M, Yamaji K, Tamura N, Makino S, Takasaki Y: Predictive value of bone destruction and duration of clinical remission for subclinical synovitis in rheumatoid arthritis patients. Mod Rheumatol, 2015; 25: 540-545.

15) Tsuru T, Tanaka Y, Kishimoto M, Saito K, Yoshizawa S, Takasaki Y, Miyamura T, Niiro $\mathrm{H}$, Morimoto S, Yamamoto J, Lledo-Garcia R, Shao J, Tatematsu S, Togo O, Koike T: Safety, pharmacokinetics, and pharmacodynamics of epratuzumab in Japanese patients with moderate-to-severe systemic lupus erythematosus: Results from a phase $1 / 2$ randomized study. Mod Rheumatol, 2016; 26: 87-93.

16) Yamazaki H, Sakai R, Koike R, Miyazaki $Y$, Tanaka M, Nanki T, Watanabe K, Yasuda S, Kurita T, Kaneko Y, Tanaka Y, Nishioka Y, Takasaki Y, Nagasaka K, Nagasawa H, Tohma S, Dohi M, Sugihara T, Sugiyama H, Kawaguchi Y, Inase N, Ochi S, Hagiyama H, Kohsaka H, Miyasaka N, Harigai M, for the PREVENT Study Group: Assessment of risks of pulmonary infection during 12 months following immunosuppressive treatment for active connective tissue diseases: a large-scale prospective cohort study. J Rheumatol, 2015; 42: 614-622.

17) Yasuda $S$, Atsumi $T$, Shimamura $S$, Ono $K$, Hiromura K, Sada K, Mori M, Takei S, Kawaguchi Y, Tamura N, Takasaki Y: Surveillance for the use of mycophenolate mofetil for adult patients with lupus nephritis in Japan. Mod Rheumatol, 2015; 25: 854-857.

\section{Dermatology and Allergology}

〈Orginal Articles〉

1) Arima $K$, Ohta $S$, Takagi A, Shiraishi $H$, Masuoka M, Ontsuka K, Suto H, Suzuki S, Yamamoto K, Ogawa M, Simmons O, Yamaguchi Y, Toda S, Aihara M, Conway SJ, Ikeda S, Izuhara K: Periostin contributes to epidermal hyperplasia in psoriasis common to atopic dermatitis. Allergol Int, 2015; 64: 41-48.

$\dagger$ 2) Smithrithee R, Niyonsaba F, Kiatsurayanon C, Ushio H, Ikeda S, Okumura K, Ogawa H: Human $\beta$-defensin-3 increases the expression of interleukin-37 through CCR6 in hyman keratinocytes. J Dermatol Sci, 2015; 77: 46-53.

3) Furusawa K, Hasegawa T, Hirasawa Y, Ikeda $\mathrm{S}$ : Mucous membrane pemphigoid with esophageal stricture treated with balloon dilatation. J Dermatol, 2015; 42: 325-326.

4) Ishii S, Hiruma M, Hayakawa $Y$, Sugita $T$, Makimura K, Hiruma M, Yoshiike T: Cutaneous Pseudallescheria boydii/Scedosporium apiospermum complex (molecular type: Scedosporium apiospermum [Clade 4]) infection: a case report and literature review of cases from Japan. Med Mycol J, 2015; 56: E25-30. 
5) Suga Y, Tsuda T, Nagai M, Sakaguchi $Y$, Jitsukawa O, Yamamoto M, Hitomi K, Yamanishi K: Lamellar ichthyosis with pseudoexon activation in the transglutaminase 1 gene. $\mathrm{J}$ Dermatol, 2015; 42: 642-645.

6) Hayashi R, Inoue A, Suga Y, Aoki J, Shimomura Y: Analysis of unique mutations in the LPAR6 gene identified in a Japanese family with autosomal recessive woolly hair/hypotrichosis: establishment of a useful assay system for LPA6. J Dermatol Sci, 2015; 78: 197-205.

7) Kamata Y, Tominaga M, Sakaguchi A, Umehara Y, Negi O, Ogawa H, Takamori K: Retinoid-related orphan receptor $\alpha$ is involved in induction of semaphorin 3A expression in normal human epidermal keratinocytes. J Dermatol Sci, 2015; 79: 84-86.

* 8) Tsutsui-Takeuchi M, Ushio H, Fukuda M, Yamada T, Niyonsaba F, Okumura K, Ogawa $\mathrm{H}$, Ikeda S: Roles of retinoic acid-inducible gene-like receptors (RLRs), Toll-like receptors (TLR) 3 and 2'-5' oligoadenylate synthetase as viral recognition receptors on human mast cells in response to viral infection. Immunol Res, 2015; 61: 240-249.

9) Kimura U, Yokoyama K, Hiruma M, Kano R, Takamori K, Suga Y: Tinea faciei caused by Trichophyton mentagrophytes (molecular type Arthroderma benhamiae) mimics impetigo: a case report and literature review of cases in Japan. Med Mycol J, 2015; 56: E1-5.

10) Okamura $K$, Abe $Y$, Fukai $K$, Tsuruta $D$, Suga Y, Nakamura M, Funasaka Y, Oka M, Suzuki N, Wataya-Kaneda M, Seishima M, Hozumi Y, Kawaguchi M, Suzuki T: Mutation analyses of patients with dyschromatosis symmetrica hereditaria: ten novel mutations of the ADAR1 gene. J Dermatol Sci, 2015; 79: 88-90.

11) Kamijo M, Wada A, Mineki R, Sakanishi T, Ikeda S: Prostaglandin E receptor 4 inhibition restores UVB-induced downregulation of ATP2A2/SERCA2 in cultured normal human keratinocytes. J Dermatol Sci, 2015; 81: $69-71$.

12) Hasegawa T, Sakamoto A, Wada A, Fukai T, Iida H, Ikeda S: Keratinocyte progenitor cells reside in human subcutoneous tissue. PLoS
One, 2015; 10: e0118402.

13) Yashiro T, Kubo M, Ogawa H, Okumura K, Nishiyama C: PU.1 suppresses th2 cytokine expression via silencing of GATA3 transcription in dendritic cells. PLoS One, 2015; 10: e0137699.

14) Yamazaki S, Nakano N, Honjo A, Hara M, Maeda K, Nishiyama C, Kitaura J, Ohtsuka Y, Okumura K, Ogawa H, Shimizu T: The transcription factor Ehf is involved in TGF$\beta$-induced suppression of $F c \varepsilon R I$ and $c$-kit expression and FceRI-mediated activation in mast cells. J Immunol, 2015; 195: 3427-3435.

15) Ando N, Nakamura $Y$, Aoki R, Ishimaru K, Ogawa H, Okumura K, Shibata S, Shimada S, Nakao A: Circadian gene clock regulates psoriasis-like-skin inflamation in mice. J Invest Dermatol, 2015; 135: 3001-3008.

16) Umehara $Y$, Kamata $Y$, Tominaga M, Niyonsaba F, Ogawa H, Takamori K: Cathelicidin LL-37 induced Semaphorin 3A expression in human epidermal keratinocytes: implications for possible application to pruritus. J Invest Dermatol, 2015; 135: 2887-2890.

17) Ishiyama $K$, Yashiro $T$, Nakano N, Kasakura K, Miura R, Hara M, Kawai F, Maeda K, Tamura N, Okumura K, Ogawa H, Takasaki Y, Nishiyama C: Involvment of PU.1 in NFATc1 promoter function in osteoclast development. Allergol Int, 2015; 64: 241-247.

18) Nakano N, Nishiyama C, Yagita H, Hara M, Motomura Y, Kubo M, Okumura K, Ogawa H: Notch signaling enhances FceRI-mediated cytokine production by mast cells through direct and indirect mechanisms. J Immunol, 2015; 194: 4535-4544.

19) Nishikado H, Fujimura T, Taka H, Mineki R, Ogawa H, Okumura K, Takai T: Cystein protease antigens cleave $\mathrm{CD} 123$, the subunit of murine IL-3 receptor, on basophils and suppress IL-3-mediated basophil expansion. Biochem Biophys Res Commun, 2015; 460: 261-266.

20) Iwabuchi K, Masuda H, Kaga N, Nakayama H, Matsumoto R, Iwahara C, Yoshizaki F, Tamaki Y, Kobayashi T, Hayakawa T, Ishii K, Yanagida M, Ogawa H, Takamori K: Properties and fuction of lactosylceramide from mouse neutrophils. Glycobiology, 2015; 25: 
655-668.

21) Suzuki S, Morimoto S, Fujishiro M, Kawasaki M, Hayakawa K, Miyashita T, Ikeda K, Miyazawa K, Yanagida M, Takamori K, Ogawa H, Sekigawa I, Takasaki Y: Inhibition of the insulin-like growth factor system is a potential therapy for rheumatoid arthritis. Autoimmunity, 2015; 48: 251-258.

〈Reviews〉

1) Hasegawa T, Ikeda S: Adipose-derived stromal/stem cells as a potential souce of skin regeneration. Stem Cell \& Translational Investigation, 2015; 2: e787.

2) Hiruma J, Ogawa Y, Hiruma M: Trichophyton tonsrans infection in Japan: epidemiology, clinical features, diagnosis and infection control. J Dermatol, 2015; 42: 245-249.

3) Iwabuchi K, Nakayama H, Oizumi A, Suga Y, Ogawa H, Takamori K: Role of ceramide from glycosphingolipids and its metabolites in immunological and inflammatory responses in human. Mediators Inflamm, 2015; 2015: 120748 .

\section{General Medicine}

$\langle$ Orginal Articles〉

1) Fujibayashi K, Yokokawa H, Gunji T, Sasabe N, Okumura M, Iijima K, Haniu T, Hisaoka T, Fukuda H: Utility of $75^{-} \mathrm{g}$ oral glucose tolerance test results and hemoglobin alc values for predicting the incidence of diabetes mellitus among middle-aged Japanese men -a large-scale retrospective cohort study performed at a single hospital. Intern Med, 2015; 54: 717-723.

* 2) Suzuki A, Uehara Y, Saita M, Inui A, Isonuma H, Naito T: Raltegravir and abacavir/lamivudine in Japanese treatment-naïve and treatment-experienced HIV-infected patients: a 48-week retrospective pilot analysis. Jpn J Infect Dis, Epub 2015 May 12.

* 3) Murai K, Culleton R, Hisaoka T, Endo H, Mita T: Global distribution of polymorphisms associated with delayed Plasmodium falciparum parasite clearance following artemisinin treatment: genotyping of archive blood samples. Parasitol Int, 2015; 64: 267-273.

4) Naito T, Torikai K, Mizooka M, Mitsumoto F, Kanazawa K, Ohno S, Morita H, Ukimura A,
Mishima N, Otsuka F, Ohyama Y, Nara N, Murakami K, Mashiba K, Akazawa K, Yamamoto K, Tanei M, Yamanouchi M, Senda S, Tazuma S, Hayashi J: Relationship between causes of fever of unknown origin and inflammation markers: a multicenter collaborative retrospective study. Intern Med, 2015; 54: 1989-1994.

5) Goto Y, Yokokawa H, Fukuda H, Naito T, Hisaoka T, Isonuma H: Body mass index and waist circumference are independent risk factors for low vital capacity among Japanese participants of a health checkup: a singleinstitution cross-sectional study. Environ Health Prev Med, 2015; 20: 108-115.

6) Hung CS, Su HW, Lee YL, Weng HW, Wang YC, Naito T, Tsubouchi A, Wang GC, Fan CK: Seroprevalence, seroconversion, and toxoplasmosis risk factors among pregnant women in Taipei city, Taiwan. Jpn J Infect Dis, 2015; 68: 312-317.

7) Fu CJ, Kao CY, Lee YL, Liao CW, Chen PC, Chuang TW, Wang YC, Chou CM, Huang YC, Naito T, Fan CK: Seroprevalence and associated risk factors of toxocariasis among college students in Taipei city, Taiwan. Iran J Parasitol, 2015; 10: 482-189.

8) Yokokawa H, Yuasa M, Sanada H, Hisaoka T, Fukuda H: Age- and sex-specific impact of health literacy on healthy lifestyle characteristics among Japanese residents in a rural community. Health, 2015; 7: 679-688.

9) Kudo N, Yokokawa H, Fukuda H, Sanada H, Miwa Y, Hisaoka T, Isonuma H: Achievement of target blood pressure levels among Japanese workers with hypertension and healthy lifestyle characteristics associated with therapeutic failure. PLoS One, 2015; 10: e0133641.

10) Hirai $Y$, Asahata-Tago S, Ainoda $Y$, Fujita T, Kikuchi K: Edwardsiella tarda bacteremia. A rare but fatal water- and foodborne infection: review of the literature and clinical cases from a single centre. Can J Infect Dis Med Microbiol, 2015; 26: 313-318.

11) Hirai $Y$, Asahata $S$, Ainoda $Y$, Fujita $T$, Miura H, Hizuka N, Kikuchi K: Candidemia diagnosed from peripheral blood smear: case report and review of literature 1954-2013. 
Mycopathologia, 2015; 180: 111-116.

12) Asahata S, Hirai $Y$, Ainoda $Y$, Fujita T, Okada Y, Kikuchi K: Fournier's gangrene caused by Listeria monocytogenes as the primary organism. Can J Infect Dis Med Microbiol, 2015; 26: 44-46.

13) Hikone M, Ainoda $Y$, Tago S, Fujita T, Hirai Y, Takeuchi K, Totsuka K: Risk factors for recurrent hospital-acquired Clostridium difficile infection in a Japanese university hospital. Clin Exp Gastroenterol, 2015; 8: 191-196.

14) Tago $S$, Hirai $Y$, Ainoda $Y$, Fujita T, Kikuchi K: Gram-negative rod bacteremia after cardiovascular surgery: Clinical features and prognostic factors. J Microbiol Immunol Infect, 2015; pii: S1684-1182 (15) 00814-2.

15) Tago S, Hirai $Y$, Ainoda $Y$, Fujita $T$, Takamori M, Kikuchi K: Perianal tuberculosis: A case report and review of the literature. World J Clin Cases, 2015; 3: 848-852.

16) Takahashi H, Kutasy B, Pes L, Paradisi F, Puri P: Decidual $\beta$-carotene-15, 15 '-oxygenase -1 and 2 (BCMO1,2) expression is increased in nitrofen model of congenital diaphragmatic hernia. Pediatr Surg Int, 2015; 31: $37-43$.

17) Takahashi T, Friedmacher F, Takahashi H, Hofmann AD, Puri P: Kif7 expression is decreased in the diaphragmatic and pulmonary mesenchyme of nitrofen-induced congenital diaphragmatic hernia. J Pediatr Surg, 2015; 50: 904-907.

\section{Clinical Pharmacology}

〈Orginal Articles〉

1) Kataoka Y, Yasuda S, Miyamoto Y, Sase K, Kosuge M, Kimura K, Yoshimasa Y, Miyazaki S; DIANA study investigators: Atheroma progression in obese early-stage diabetic Japanese patients in response to glycemic control: sub-analysis from the DIANA study. J Atheroscler Thromb, 2015; 22: 697-705.

〈Reviews〉

1) IMDRF Registry Working Group (MarinacDabic D, Normand S-L, Sedrakyan A, Tscheng J, Handa N, Murakami M, Sase K, et al.): Patient Registy, Essential Principles. Proposed Document (http://www.imdrf.org/ consultations/cons-essential-principles- 151124 . asp). October 2, 2015. Accessed July 5, 2017.

\section{Sports Medicine}

〈Orginal Articles〉

1) Yaginuma $S$, Sakuraba K, Kadoya H, Koibuchi E, Matsukawa T, Ito H, Yokoyama K, Suzuki Y: Early bedtime associated with the salutary breakfast intake in Japanese nursery school children. Int Med J, 2015; 22: 30-32.

2) Suzuki Y, Shimizu T, Ota M, Hirata R, Sato K, Tamura Y, Imanishi A, Watanabe M, Sakuraba K: Different training status may alter the continuous blood glucose kinetics in selfpaced endurance running. Exp Ther Med, 2015; 10: 978-982.

* 3) Wada Y, Sakuraba K, Kubota A: Effect of the long-term care prevention project on the motor functions and daily life activities of the elderly. J Phys Ther Sci, 2015; 27: 199-203.

4) Ishikawa K, Omori K, Obinata M, MshimaK, Osaka H, Oode Y, Kubota A, Sakuraba K, Yanagawa Y: A field survey of spinal cord injury in bodybooaders. Am J Emerg Med, 2015; 33: 112-113.

5) Tominaga S, Sakuraba K, Usui F: The effects of changes in the sagittal plane alignment of running-specific transtibial prostheses on ground reaction forces. J Phys Ther Sci, 2015; 27: 1347-1351.

\section{Epidemiology and Environmental Health}

$\langle$ Orginal Articles〉

1) Hosokawa M, Tsunoda M, Liu Y, Nakano K, Itai K, Tsunoda H, Yokoyama K, Aizawa Y: The immunotoxic effects of fluoride on mice after subacute administration by evaluating cytokine mRNA expressions in splenoceytes. Fluoride, 2015; 48: 329-337.

2) Itoh, H, Takamura M, Yokoyama K: Postgraduate psychiatry training and associated greater self-rated competence in primary care physicians in internal medicine in managing mental health disorders in Japan. Juntendo Medical Journal, 2015; 61: 508-516.

3) Kano Y, Tohyama M, Aihara M, Matsukura S, Watanabe H, Sueki H, Iijima M, Morita E, Niihara H, Asada H, Kabashima K, Azukizawa H, Hashizume H, Nagao K, Takahashi H, 
Abe R, Sotozono C, Kurosawa M, Aoyama Y, Chu CY, Chung WH, Shiohara T: Sequelae in 145 patients with drug-induced hypersensitivity syndrome/drug reaction with eosinophilia and systemic symptoms: survey conducted by the Asian Research Committee on Severe Cutaneous Adverse Reactions (ASCAR). Journal of Dermatology, 2015; 42: 276-282.

4) Kimura E, Kubo K, Matsuyoshi C, Benner S, Hosokawa M, Endo T, Ling W, Kohda M, Yokoyama K, Nakajima, K, Kakeyama M, Tohyama C: Developmental origin of abnormal dendritic growth in the mouse brain induced by in utero disruption of aryl hydrocarbon receptor signaling. Neurotoxicology and Teratology, 2015; 52: 42-50.

5) Yaginuma S, Sakuraba K, Kadoya H, Koibuchi E, Matsukawa T, Ito H, Yokoyama K, Suzuki Y: Early bedtime associated with the salutary breakfast intake in Japanese nursery school children. International Medical Journal, 2015; 22: 30-32.

6) Vigeh M, Yokoyama K, Matsukawa T, Shinohara A, Ohtani K: The relation of maternal blood arsenic to anemia during pregnancy. Women \& Health, 2015; 55: 42-57.

7) Nishioka E, Hirayama S, Ueno T, Matsukawa T, Vigeh M, Yokoyama K, Makino S, Takeda S, Miida T: Relationship between maternal thyroid-stimulating hormone (TSH) elevation during pregnancy and low birth weight: a longitudinal study of apparently healthy urban Japanese women at very low risk. Early Hum Dev, 2015; 91: 181-185.

8) Nishina K, Yokoyama K, Matsukawa $T$, Nishioka E, Iijima S, Kudou A, Kitamura F: Factors affecting employment status of home caregivers of elderly people. Juntendo Medical Journal, 2015; 61: 136-141.

9) Yamanobe $Y$, Nagahara N, Matsukawa T, Ito T, Niimori-Kita K, Chiba M, Yokoyama K, Takizawa T: Sex differences in shotgun proteome analyses for chronic oral intake of cadmium in mice. PLoS One, 2015; 10: e0121819.

10) Watanabe K, Yokoyama K, Furukawa TA: Reliability and validity of the Japanese version of the coping inventory for adults for stressful situations in healthy people. Psychol Rep, 2015; 116: 447-469.

11) Uno $Y$, Uchiyama $T$, Kurosawa M, Aleksic B, Ozaki N: Early exposure to the combined measles-mumps-rubella vaccine and thimerosal-containing vaccines and risk of autism spectrum disorder. Vaccine, 2015; 33: 25112516.

12) Kiyohara C, Washio M, Horiuchi T, Takahashi H, Tada Y, Kobashi G, Asami T, Ide S, Atsumi T; The Kyushu Sapporo SLE (KYSS) Study Group (Inaba Y): Dietary patterns and the risk of systemic lupus erythematosus in a Japanese population: the Kyushu Sapporo SLE (KYSS) study. International Medical Journal, 2015; 22: 110-115.

13) Lin $Y$, Nishiyama T, Kurosawa M, Tamakoshi A, Kubo T, Fujino Y, Kikuchi S; JACC Study Group: Association between shift work and the risk of death from biliary tract cancer in Japanese men. BMC Cancer, 2015; 15: 757.

14) Konuma H, Hirose H, Yokoyama K: Relationship of the Japanese translation of the profile of mood states second edition (POMS2 ${ }^{\circledR}$ ) to the first edition $\left(\mathrm{POMS}^{\circledR}\right)$. Juntendo Medical Journal, 2015; 61: 517-519.

15) Matsukawa T, Hasegawa H, Goto H, Shinohara Y, Shinohara A, Omori Y, Ichida K, Yokoyama K: Evaluation of the metabolic chiral inversion of $\mathrm{D}$-selenomethionine in rats by stable isotope dilution gas chromatography-mass spectrometry. J Pharm Biomed Anal, 2015; 116: 59-64.

16) Matsukawa T, Yokoyama K, Itoh H: Ocular irritation from product of pesticide degradation among workers in a seed warehouse. Industrial Health, 2015; 53: 95-99.

\section{Psychiatry and Behavioral Science}

〈Orginal Articles〉

1) Hirano A, Ohara T, Takahashi A, Aoki M, Fuyuno Y, Ashikawa K, Morihara T, Takeda M, Kamino K, Oshima E, Okahisa Y, Shibata $\mathrm{N}$, Arai H, Akatsu H, Ikeda M, Iwata N, Ninomiya T, Monji A, Kitazono T, Kiyohara Y, Kubo M, Kanba S: A genome-wide association study of late-onset Alzheimer's disease in a Japanese population. Psychiatr Genet, 2015; 25: 139-146. 
2) Sato K, Kirino E, Tanaka S: A voxel-based morphometry study of the brains of university students majoring in music and in other non-music disciplines. Behavioural Neurol, 2015; 2015: 274919.

* 3) Takeda M, Ohnuma T, Takeuchi M, Katsuta N, Maeshima H, Takebayashi Y, Higa M, Nakamura T, Nishimon S, Sannohe T, Hotta Y, Hanzawa R, Higashiyama R, Shibata N, Gohda T, Suzuki Y, Yamagishi S, Tomino Y, Arai H: Altered serum glyceraldehydederived advanced glycation end product (AGE) and soluble AGE receptor levels indicate carbonyl stress in patients with schizophrenia. Neurosci Lett, 2015; 593: 51-55.

4) Kondo D, Hino H, Shibuya K, Fujisawa K, Kosaka K, Hirayasu Y, Yamamoto R, Kasanuki K, Minegishi M, Sato K, Hosokawa M, Arai T, Arai H, Iseki E: An autopsied case of corticobasal degeneration showing severe cerebral atrophy over a protracted disease course of 16 years. Neuropathology, 2015; 35: 280-288.

5) Nagata T, Kobayashi N, Ishii J, Shinagawa S, Nakayama R, Shibata N, Kuerban B, Ohnuma T, Kondo K, Arai H, Yamada H, Nakayama K: Association between DNA methylation of the BDNF promoter region and clinical presentation in Alzheimer's disease. Dement Geriatr Cogn Dis Extra, 2015; 5: 64-73.

6) Shibata N, Nagata T, Tagai K, Shinagawa S, Ohnuma T, Kawai E, Kasanuki K, Shimazaki H, Toda A, Tagata Y, Nakada T, Nakayama K, Yamada H, Arai H: Association between the catechol-O-methyltransferase polymorphism Val158Met and Alzheimer's disease in a Japanese population. Int J Geriatr Psychiatry, 2015; 30: 927-933.

* 7) Nakamura T, Ohnuma T, Hanzawa R, Takebayashi Y, Takeda M, Nishimon S, Sannohe T, Katsuta N, Higashiyama R, Shibata N, Arai H: Associations of common copy number variants in glutathione S-transferase mu 1 and D-dopachrome tautomerase-like protein genes with risk of schizophrenia in a Japanese population. Am J Med Genet B Neuropsychiatr Genet, 2015; 168: 630-636.

8) Chiba Y, Fujishiro H, Ota K, Kasanuki K, Arai H, Hirayasu Y, Sato K, Iseki E: Clinical profiles of dementia with Lewy bodies with and without Alzheimer's disease-like hypometabolism. Int J Geriatr Psychiatry, 2015; 30: $316-323$.

9) Yoshita M, Arai H, Arai H, Arai T, Asada T, Fujishiro H, Hanyu H, Iizuka O, Iseki E, Kashihara K, Kosaka K, Maruno H, Mizukami K, Mizuno Y, Mori E, Nakajima K, Nakamura H, Nakano S, Nakashima K, Nishio Y, Orimo S, Samuraki M, Takahashi A, Taki J, Tokuda T, Urakami K, Utsumi K, Wada K, Washimi Y, Yamasaki J, Yamashina S, Yamada M: Diagnostic accuracy of 123I-meta-iodobenzylguanidine myocardial scintigraphy in dementia with Lewy bodies: a multicenter study. PLoS One, 2015; 10: e0120540.

*10) Inoue T, Kohno K, Baba H, Takeshima M, Honma H, Nakai Y, Suzuki T, Hatano K, Arai H, Matsubara S, Kusumi I, Terao T: Does temperature or sunshine mediate the effect of latitude on affective temperaments? A study of 5 regions in Japan. J Affect Disord, 2015; 172: 141-145.

11) Samara MT, Leucht C, Leeflang MM, Anghelescu I, Ascher-Svanum H, Chung Y, Crespo-Facorro B, Elkis H, Hatta K, Giegling I, Kane JM, Kayo M, Lambert M, Lin C, Möller H, Pelayo-Teran JM, Riedel M, Rujescu D, Schimmelmann BG, Serretti A, Zhao JJ, Correll CU, Leucht S: Early improvement as a predictor of later response to antipsychotics in Schizophrenia: a diagnostic test review. Am J Psychiatry, 2015; 172: 617-629.

12) Häuser $W$, Ablin J, Fitzcharles MA, Littlejohn G, Luciano JV, Usui C, Walitt B: Fibromyalgia. Nat Rev Dis Primers, 2015; 1: 15022.

*13) Kawai E, Shibata N, Nagata T, Shinagawa S, Tagai K, Ohnuma T, Shimazaki H, Toda A, Kasanuki K, Takayama T, Suzuki A, Nakayama K, Yamada H, Arai H: Genetic association between KIBRA polymorphism and Alzheimer's disease with in a Japanese population. Neuromolecular Med, 2015; 17: 209.

14) Kasanuki K, Iseki E, Fujishiro $H$, Ando S, Sugiyama H, Kitazawa M, Chiba Y, Sato K, Arai H: Impaired heart rate variability in patients with dementia with Lewy bodies: efficacy of electrocardiogram as a supporting 
diagnostic marker. Parkinsonism Relat Disord, 2015; 21: 749-754.

15) Murphy KE, Gysbers AM, Abbott SK, Spiro AS, Furuta A, Cooper A, Garner B, Kabuta T, Halliday GM: Lysosomal-associated membrane protein 2 isoforms are differentially affected in early Parkinson's disease. Mov Disord, 2015; 30: 1639-1647.

16) Suenaga M, Furuta A, Wakabayashi K, Saibara T, Matsunaga Y: Monocytic elastasemediated apolipoprotein-E degradation: Potential involvement of microglial elastaselike proteases in apolipoprotein-E proteolysis in brains with Alzheimers disease. Biochim Biophys Acta, 2015; 1854: 1010-1018.

17) Kawano R, Ishida M, Kimura E, Matsumoto H, Arai H: Pilot intervention study of a low-salt diet with monomagnesium di-L-glutamate as an umami seasoning in psychiatric inpatients. Psychogeriatrics, 2015; 15: 38-42.

18) Fujishiro H, Nakamura S, Sato K, Iseki E: Prodromal dementia with Lewy bodies. Geriatr Gerontol Int, 2015; 15: 817-826.

19) Furuta A, Kikuchi H, Fujita H, Yamada D, Fujiwara Y, Kabuta T, Nishino I, Wada K, Uchiyama Y: Property of lysosomal storage disease associated with midbrain pathology in the central nervous system of Lamp-2deficient mice. Am J Pathol, 2015; 185: 17131723.

20) Iwanami T, Maeshima $H$, Baba H, Satomura E, Namekawa Y, Shimano T, Suzuki T, Arai $\mathrm{H}$ : Psychomotor agitation in major depressive disorder is a predictive factor of moodswitching. J Affect Disord, 2015; 170: 185189.

21) Hatta K, Kishi $Y$, Wada K: Ramelteon for delirium in hospitalized patients. JAMA, 2015; 314: 1071-1072.

* 22) Nomoto H, Baba H, Satomura E, Maeshima H, Takebayashi N, Namekawa Y, Suzuki T, Arai H: Serum brain-derived neurotrophic factor levels and personality traits in patients with major depression. BMC Psychiatry, 2015; 4: 33.

23) Kirino E, Imagawa $H$, Goto $T$, Montgomery W: Socio-demographics, comorbidities, healthcare utilizatiom and work productivity in Japanese patients with adult ADHD. PLoS
One, 2015; 10: e0132233.

24) Asano T, Baba H, Kawano R, Takei H, Maeshima H, Takahashi Y, Suzuki T, Arai H: Temperament and character as predictors of recurrence in remitted patients with major depression: a 4-year prospective follow-up study. Psychiatry Res, 2015; 225: 322-325.

25) Fujita H, Yagishita N, Aratani S, Saito-Fujita T, Morota S, Yamano Y, Hansson MJ, Inazu M, Kokuba H, Sudo K, Sato E, Kawahara K, Nakajima F, Hasegawa D, Higuchi I, Sato T, Araya N, Usui C, Nishioka K, Nakatani Y, Maruyama I, Usui M, Hara N, Uchino H, Elmér E, Nishioka K, Nakajima T: The E3 ligase synoviolin controls body weight and mitochondrial biogenesis through negative regulation of PGC-1B. EMBO J, 2015; 34: 1042-1055.

26) Arai H, Suzuki H, Yoshiyama T: Vanutide cridificar and the QS-21 adjuvant in Japanese subjects with mild to moderate Alzheimer's disease: results from two phase 2 studies. Curr Alzheimer Res, 2015; 12: 242-254.

27) Ota K, Murayama N, Kasanuki K, Kondo D, Fujishiro H, Arai H, Sato K, Iseki E: Visuoperceptual assessments for differentiating dementia with Lewy bodies and Alzheimer's disease: illusory contours and other neuropsychological examinations. Arch Clin Neuropsychol, 2015; 30: 256-263.

〈Reviews〉

1) Kirino E: Use of aripiprazole for delirium in the elderly: a short review. Psychogeriatrics, 2015; 15: 75-84.

2) Hatta K: Practical pharmacotherapy for acute schizophrenia patients. Psychiatry Clin Neurosci, 2015; 69: 674-685.

3) Endo Y, Furuta A, Nishino I: Danon disease: a phenotypic expression of LAMP-2 deficiency. Acta Neuropathol, 2015; 129: 391398.

\section{Forensic Medicine}

〈Orginal Articles〉

1) Nakanishi H, Shojo H, Ohmori T, Hara M, Takada A, Adachi N and Saito K: A novel method for sex determination by detecting the number of $\mathrm{X}$-chromosomes. Int J Legal Med, 2015; 129: 23-29. 
2) Takahashi S, Takada A, Saito K, Hara M, Yoneyama K, Nakanishi H, Takahashi K, Moriya T, Funayama M: Sudden death of a child from myocardial infarction due to arteritis of the left coronary trunk. Legal Med (Tokyo), 2015; 17: 39-42.

3) Hara M, Nakanishi H, Takahashi S, Nagai A, Yamamoto T, Yoneyama K, Saito K, Takada A: Relationship between DNA degradation ratios and the number of loci detectable by STR kits in extremely old seminal stain samples. Legal Med (Tokyo), 2015; 17: 391393.

* 4) Nakahara H, Fujii K, Kitayama T, Sekiguchi K, Nakanishi H, Saito K: Approaches for identifying multiple-SNP haplotype blocks for use in human identification. Legal Med (Tokyo), 2015; 17: 415-420.

5) Takada A, Saito K, Murai T, Kurosaki K, Kurihara K, Hamamatsu A: Right ventricular free wall dissection as a rupture tract in left ventricular rupture during acute myocardial infarction. Legal Med (Tokyo), 2015; 17: 525-531.

6) Takahashi S, Takada A, Saito K, Hara M, Yoneyama K, Nakanishi H: Fatal anaphylaxis associated with the gadolinium-based contrast agent gadoteridol (ProHanceR). J Investig Allergol Clin Immunol, 2015; 25: 365-384

7) Saito K, Kurosu A, Takada A, Hasegawa I, Mori S: "Black esophagus" associated with a poisoning of an antipyretic analgesic containing caffeine. Res Pract Forens Med, 2015; 58: $252-253$

\section{Public Health}

〈Orginal Articles〉

1) Minematsu K, Kawabuchi R, Okazaki H, Tomita H, Tobina T, Tanigawa T, Tsunawake N: Physical activity cut-offs and risk factors for preventing child obesity in Japan. Pediatr Int, 2015; 57: 131-136.

2) Saito I, Hitsumoto S, Maruyama K, Nishida W, Eguchi E, Kato T, Kawamura R, Takata Y, Onuma H, Osawa H, Tanigawa T: Heart rate variability, insulin resistance, and insulin sensitivity in Japanese adults: the Toon Health Study. J Epidemiol, 2015; 25: 583-591.
* 3) Higuchi K, Saito I, Maruyama K, Eguchi E, Mori H, Tanno S, Sakurai S, Kishida T, Nishida W, Osawa H, Tanigawa T: Associations of serum $\beta$-carotene and retinol concentrations with insulin resistance: the Toon Health Study. Nutrition, 2015; 31: 975-980.

4) Maruyama K, Nishioka S, Miyoshi N, Higuchi K, Mori H, Tanno S, Tomooka K, Eguchi E, Furukawa S, Saito I, Sakurai S, Nishida W, Osawa H, Tanigawa T: The impact of masticatory ability as evaluated by salivary flow rates on obesity in Japanese: the Toon Health Study. Obesity (Silver Spring), 2015; 23: $1296-1302$.

5) Higaki M, Wada H, Mikura S, Yasutake T, Nakamura M, Niikura M, Kobayashi F, Kamma H, Kamiya S, Goto H, Ito K, Barnes PJ, Takizawa H: Interleukin-10 modulates pulmonary neutrophilic inflammation induced by cigarette smoke exposure. Exp Lung Res, 2015; 41: 525-534.

6) Nakamura M, Wada H, Honda K, Nakamoto K, Inui T, Sada M, Watanabe M, Takata S, Yokoyama T, Saraya T, Kurai D, Ishii H, Goto H, Kamma H, Takizawa H: Clarithromycin ameliorates pulmonary inflammation induced by short term cigarette smoke exposure in mice. Pulm Pharmacol Ther, 2015; 35: 60-66.

7) Sata M, Yamagishi K, Sairenchi T, Ikeda A, Irie $\mathrm{F}$, Watanabe $\mathrm{H}$, Iso H, Ota H: Impact of caregiver type for 3-year-old children on subsequent between-meal eating habits and being overweight from childhood to adulthood: a 20-year follow-up of the Ibaraki Children's Cohort (IBACHIL) Study. J Epidemiol, 2015; 25: 600-607.

8) Gijsberts CM, Groenewegen KA, Hoefer IE, Eijkemans MJ, Asselbergs FW, Anderson TJ, Britton AR, Dekker JM, Engström G, Evans GW, de Graaf J, Grobbee DE, Hedblad B, Holewijn S, Ikeda A, Kitagawa K, Kitamura A, de Kleijn DP, Lonn EM, Lorenz MW, Mathiesen EB, Nijpels G, Okazaki S, O'Leary DH, Pasterkamp G, Peters SA, Polak JF, Price JF, Robertson C, Rembold CM, Rosvall M, Rundek T, Salonen JT, Sitzer M, Stehouwer CD, Bots ML, den Ruijter HM: Race/ethnic differences in the associations of the framingham risk factors with carotid IMT and 
cardiovascular events. PLoS One, 2015; 10: e0132321.

9) Honjo K, Iso H, Ikeda A, Fujino Y, Tamakoshi A; JACC Study Group: Employment situation and risk of death among middle-aged Japanese women. J Epidemiol Community Health, 2015; 69: 1012-1017.

10) Honjo K, Iso H, Nakaya T, Hanibuchi T, Ikeda A, Inoue M, Sawada N, Tsugane S; Japan Public Health Center-based Prospective Study Group: Impact of neighborhood socioeconomic conditions on the risk of stroke in Japan. J Epidemiol, 2015; 25: 254-260.

11) Eikendal AL, Groenewegen KA, Anderson TJ, Britton AR, Engström G, Evans GW, de Graaf J, Grobbee DE, Hedblad B, Holewijn S, Ikeda A, Kitagawa K, Kitamura A, Lonn EM, Lorenz MW, Mathiesen EB, Nijpels G, Dekker JM, Okazaki S, O'Leary DH, Polak JF, Price JF, Robertson C, Rembold CM, Rosvall M, Rundek T, Salonen JT, Sitzer M, Stehouwer CD, Hoefer IE, Peters SA, Bots ML, den Ruijter HM; USE-IMT Project Group: Common carotid intima-media thickness relates to cardiovascular events in adults aged $<45$ years. Hypertension, 2015; 65: 707-713.

12) Kawabata-Shoda E, Charvat H, Ikeda A, Inoue M, Sawada N, Iwasaki M, Sasazuki S, Shimazu T, Yamaji T, Kimura H, Masuda S, Tsugane S: Trends in cancer prognosis in a population-based cohort survey: can recent advances in cancer therapy affect the prognosis? Cancer Epidemiol, 2015; 39: 97-103.

13) Svensson T, Inoue M, Sawada N, Iwasaki M, Sasazuki S, Shimazu T, Yamaji T, Ikeda A, Kawamura N, Mimura M, Tsugane S; Japan Public Health Centre-based prospective Study Group: The association between complete and partial non-response to psychosocial questions and suicide: the JPHC Study. Eur J Public Health, 2015; 25: 424-430.

14) Hanibuchi $T$, Nakaya $T$, Honjo $K$, Ikeda $A$, Iso $\mathrm{H}$, Inoue $\mathrm{M}$, Sawada $\mathrm{N}$, Tsugane S; Japan Public Health Center-based Prospective Study Group: Neighborhood contextual factors for smoking among middle-aged Japanese: a multilevel analysis. Health Place, 2015; 31: 17-23.

15) Saito I, Maruyama K, Eguchi E: C-reactive protein and cardiovascular disease in East Asians: a systematic review. Clin Med Insights Cardiol, 2015; 8 (Suppl 3): 35-42.

16) Maruyama K, Kokubo Y, Yamanaka $T$, Watanabe M, Iso H, Okamura T, Miyamoto Y: The reasonable reliability of a self-administered food frequency questionnaire for an urban, Japanese, middle-aged population: the Suita Study. Nutr Res, 2015; 35: 14-22.

17) Yokokawa H, Yuasa M, Sanada H, Hisaoka T, Fukuda H: Age- and sex-specific impact of health literacy on healthy lifestyle characteristics among Japanese residents in a rural community. Health, 2015; 7: 679-688.

18) Yuasa M, Sirayama $Y$, Kigawa $M$, Chaturanga I, Mizoue T, Kobayashi H: A health promoting schools (HPS) program among primary and secondary school children in Southern Province, Sri Lanka: a qualitative study on the program's effects on the school children, parents, and teachers. Journal of International Health, 2015; 30: 93-101.

19) Yuasa M, Sirayama $Y$, Osato K, Miranda C, Condore J, Siles R: Cross-sectional analysis of self-efficacy and social capital in a community-based healthy village project in Santa Cruz, Bolivia. BMC International Health \& Human Rights, 2015; 15: 15.

20) Minematsu K, Kawabuchi R, Okazaki H, Tomita H, Tobina T, Tanigawa T, Tsunawake N: Physical activity cut-offs and risk factors for preventing child obesity in Japan. Pediatr Int, 2015; 57: 131-136.

〈Books〉

1) Wada $H$, Inui $T$, Nakamoto $K$, Sada $M$, Nakamura M, Takizawa H: A Pivotal Role of Nrf2 in Airway Inflammatory Responses Inducedby Diesel Exhaust in Asthma: Mechanisms and Their Application to Clinical Interventions. In: Li Y-J, Umezawa M, Takizawa H, Takeda K, Kawada T, eds. PM2.5: Role of Oxidative Stress in Health Effects and Prevention Strategy. USA: Nova Science Publishers, 2015; 59-72.

\section{Medical Education}

〈Orginal Articles〉

$\dagger 1$ ) He S, Kempe K, Tomiki Y, Nishizuka M, Suzuki T, Dambara T, Okada T: Correlations 
between entrance examination scores and academic performance following admission. Juntendo Medical Journal, 2015; 61: 142-148.

2) He S, Kempe K, Tomiki Y, Nishizuka M, Suzuki T, Okada T, Dambara T: Correlation between scores in clinical resident training and academic performance prior to/following admission to the school. Juntendo Medical Journal, 2015; 61: 503-507.

3) Sekiya M, Yoshimi K, Muraki K, Iwakami S, Togo S, Tamaki S, Dambara T, Takahashi K:
Do respiratory comorbidities limit the diagnostic usefulness of ultrasound-guided needle aspiration for subpleural lesions? Respiratory Investigation, 2015; 53: 98-103.

4) Tomiki Y, Suzuki T, Tambara K, Nojima M, Sumiyoshi M, Isobe Y, Ishiga D, Shimizu T, Kobayashi H, Kaneko K, Dambara T: Residents' career choice process -a questionnaire survey after the revision of clinical training systems -second report-. Juntendo Medical Journal, 2015; 61: 57-61. 